\section{COPD-Exazerbation: Eosinophile-gesteuerte Kortikoidgabe verkürzt Therapiedauer}

Sivapalan P et al. Eosinophil-guided corticosteroid therapy in patients admitted to hospital with COPD exacerbation (CORTICO-COP): a multicentre, randomised, controlled, openlabel, non-inferiority trial. Lancet Respir Med 2019; doi:10.1016/S2213-2600(19)30176-6

Eine mehrtägige Behandlung mit systemischen Kortikosteroiden zählt zum Standard bei akuten Exazerbationen einer COPD. Da diese allerdings das Immunsystem schwächen und zu nachteiligen Nebenwirkungen führen können, werden Wege zur besseren Therapiesteuerung und Dosisreduktion gesucht. Sivapalan et al. haben daher nun eine an der Eosinophilenzahl orientierte Dosisanpassung mit dem Standardvorgehen mit fixer Dosierung über 5 Tage verglichen.

Eine akute Exazerbation bei Patienten mit chronisch obstruktiver Lungenerkrankung (COPD) geht mit erhöhter Morbidität, Mortalität und einer schlechteren Lebensqualität einher. Weltweit hat sich die systemische Therapie mit Kortikosteroiden über bis zu 5 Tage als Standard etabliert. Dieser Therapieansatz kann aber die stetige Abnahme der Lungenfunktion nicht aufhalten und führt bei vielen Patienten zu teils schwerwiegenden Nebenwirkungen wie osteoporotisch bedingten Frakturen, Nebenniereninsuffizienz sowie Verschlechterung eines vorbestehenden Diabetes mellitus.

Vor diesem Hintergrund werden Biomarker und Methoden zur individuellen Therapiesteuerung mit Ziel einer Dosisreduktion im Vergleich zum aktuellen Standard gesucht. Da sich systemische Kortikosteroide auch auf die Anzahl der Eosinophilen im Sinne einer Verminderung auswirken können und ein Großteil der COPDPatienten eine erhöhte Eosinophilenzahl vorweist, könnte eine Dosissteuerung auf Basis dieses Laborwertes eine Lösung sein. Sivapalan und Team haben daher nun eine randomisierte, kontrollierte und offene Nichtunterlegenheitsstudie zum Thema durchgeführt, und dabei eine Eosinophile-gesteuerte Kortikoidtherapie mit dem Standardvorgehen verglichen.

Die sogenannte CORTICO-COP-Studie fand zwischen August 2016 und September 2018 an 3 spezialisierten Zentren in Dänemark statt. Die Studienautoren stellten zunächst die Hypothese auf, dass eine Dosisanpassung der systemischen Kortikosteroide dem Standardvorgehen mit fixer Dosis nicht unterlegen sei und zu einer kürzeren Behandlungsdauer führen würde. Geeignete Patienten sollten folgende Kriterien erfüllen:

- Teilnahme an der Studie innerhalb von 24 Stunden nach Diagnose,

- Alter ab 40 Jahren,

- COPD-Diagnose,

- akute Exazerbation,

- fortgeschrittenes Krankheitsstadium.

Die wichtigsten Ausschlusskriterien waren eine Asthma-Diagnose, eine Lebenserwartung von unter 30 Tagen, eine sehr schwere Exazerbation mit Notwendigkeit einer invasiven Beatmung sowie eine Kortisonallergie.

Die Forscher bildeten 2 Studiengruppen:

- Interventionsgruppe (IG): $80 \mathrm{mg}$ Methylprednisolon intravenös an Tag 1 und 37,5 mg Prednisolon oral für maximal 4 Tage, sofern die Eosinophilenzahl am entsprechenden Tag über $0,3 \times 10^{9}$ Zellen pro L lag.

- Kontrollgruppe (KG): 80 mg Methylprednisolon intravenös an Tag 1 gefolgt von $37,5 \mathrm{mg}$ Prednisolon oral einmal täglich an Tag 2 bis 5 .

Als primären klinischen Endpunkt definierten die Forscher die Lebenstage und Krankenhaus-freien Tage bis 14 Tage nach der Entlassung. Sekundäre Endpunkte waren u.a. Therapieversagen innerhalb von 30 Tagen, erneutes Auftreten einer akuten Exazerbation oder Todesfall innerhalb von 30 Tagen, Zeit bis zum Rückfall, Mortalität bis 30 Tage nach Therapiebeginn, Hyperglykämie sowie Verschlechterung eines Diabetes.

\section{Kürzere Therapiedauer}

1363 Patienten wurden gescreent, 318 nahmen an der Studie teil, 159 Patienten pro Gruppe. Sie waren im Durchschnitt 75 Jahre alt, 46\% (IG) bzw. 44\% (KG) von ihnen männlich, 34\% bzw. 31\% waren aktive Raucher. Für den primären klinischen Endpunkt, die Lebenstage sowie die Krankenhaus-freien Tage bis zum 14.Tag nach Entlassung, konnten die Studienautoren keinen signifikanten Gruppenunterschied feststellen. Ein Therapieversagen nach 30 Tagen trat bei $26 \%$ der Patienten der Interventionsgruppe und bei $26 \%$ der Patienten der Kontrollgruppe auf.

Im selben Zeitraum waren 6\% der IG-Patienten, aber nur $4 \%$ der KG-Patientin verstorben. Die mediane Behandlungsdauer war bei der Interventionsgruppe mit 2 Tagen im Vergleich zu 5 Tagen bei der Kontrollgruppe deutlich geringer, dieser Unterschied war signifikant. Die Studienautoren sehen auf Basis dieser Ergebnisse ihre Hypothese bestätigt, empfehlen aber dennoch weitere Studien, da sich die Intervention bei einigen sekundären Endpunkten negativ ausgewirkt hatte.

FAZIT

In dieser randomisierten, klinischen Studie mit COPD-Patienten mit akuter Exazerbation war eine Eosinophilegesteuerte systemische Therapie mit Kortikosteroiden dem Standardvorgehen hinsichtlich des Überlebens und der krankenhausfreien Tage bis Tag 14 nach Therapiebeginn nicht unterlegen. Da die Behandlungsdauer bei dieser Therapieanpassung zwar kürzer ausfiel, aber auch für Nachteile bei sekundären Endpunkten sorgte, empfehlen die Autorinnen/Autoren weitere Studien.

Dipl.-Psych. Annika Simon, Hannover 